\title{
Prevalence and distribution of cervical high-risk human papillomavirus and cytological abnormalities in women living with HIV in Denmark - the SHADE
}

Kristina Thorsteinsson ${ }^{1,12^{*}}$ (D), Merete Storgaard ${ }^{2}$, Terese L. Katzenstein ${ }^{3,4}$, Steen Ladelund ${ }^{5}$, Frederikke Falkencrone Rønsholt ${ }^{3}$, Isik Somuncu Johansen ${ }^{6}$, Gitte Pedersen ${ }^{7}$, Lailoma Hashemi ${ }^{8}$, Lars Nørregård Nielsen ${ }^{9}$, Lisbeth Nilas ${ }^{4,10}$, Niels Obel ${ }^{3,4}$, Jesper Bonde ${ }^{11}$ and Anne-Mette Lebech ${ }^{1,4}$

\begin{abstract}
Background: Women living with HIV (WLWH) are at increased risk of persistent human papillomavirus (HPV) infection, cervical dysplasia and cervical cancer compared with women from the general population (WGP). We assessed the prevalence and distribution of cervical high-risk (hr) HPV infection and cytological abnormalities in WLWH compared with WGP in Denmark. Predictors of HPV and cytological abnormalities were estimated in WLWH.

Methods: WLWH consecutively enrolled in the Study on HIV, cervical Abnormalities and infections in women in Denmark (SHADE) in 2011 and were examined for cervical HPV and cytological abnormalities.

WLWH were matched on age and prior cytological findings with WGP from an earlier study. HIV demographics were retrieved from the nationwide Danish HIV Cohort Study. Logistic regression was used to estimate predictors of hrHPV and cytological abnormalities.
\end{abstract}

Results: Of 334 included WLWH $26.4 \%$ were positive for hrHPV as opposed to $16.6 \%$ WGP $(p<0.0001)$. WLWH had a higher number of multiple infections ( $>1$ h genotype present) (38.5 \% versus $25.7 \%, p=0.030$ ). Hr genotypes in descending order of frequency were HPV58 (7.1\%), 52 (5.4\%), and 16 (4.8 \%) in WLWH versus HPV16 (4.1\%), 52 $(2.8 \%)$ and $58(2.4 \%)$ in WGP. Predictors of hrHPV in WLWH were short duration of HAART (adjusted OR per year 0.90 (95\% Cl 0.84-0.96)), AIDS prior to inclusion (adjusted OR 3.61 (95\% Cl 1.75-7.46)), $\geq 5$ lifetime sexual partners (adjusted OR 2.20 (95\% Cl 1.08-4.49)), sexual debut <16 years of age (adjusted OR 2.05 (95\% Cl 1.03-4.10)) and CD4 $<350$ cells/ $\mu \mathrm{L}$ (adjusted OR 2.53 (95\% Cl 1.20-5.40)). Cytological abnormalities were prevalent in $10.4 \%$ vs. $5.2 \%(p=0.0003)$ of WLWH and WGP. In WLWH with hrHPV, short duration of HAART predicted cervical dysplasia (adjusted OR per year 0.83 (95\% Cl 0.71-0.97)).

Conclusions: WLWH presented with more cervical hrHPV infections and cytological abnormalities, and a different distribution of hrHPV genotypes compared with WGP. Cervical hrHPV and cytological abnormalities were predicted by short duration of HAART.

Keywords: Women living with HIV, High-risk human papillomavirus, Cervical cytological abnormalities, HPV vaccine, Highly active antiretroviral therapy

\footnotetext{
*Correspondence: Kristina.thorsteinsson@gmail.com

'Department of Infectious Diseases, Hvidovre, Copenhagen University

Hospital, Copenhagen, Denmark

${ }^{12}$ Department of Infectious Diseases, Hvidovre Hospital, Kettegaards Allé 30,

2650 Hvidovre, Denmark

Full list of author information is available at the end of the article
} 


\section{Background}

Human papillomavirus (HPV) is the most common sexually transmitted disease (STD) [1] with a lifetime prevalence of up to $80 \%$ [2]. Essentially, all cases of cervical cancer (CC) are associated with high-risk HPV (hrHPV) [3] and worldwide approximately half a million women develop $\mathrm{CC}$ each year [4]. Women living with HIV (WLWH) are at increased risk of persistent HPV infection, cervical dysplasia and CC compared with women from the general population (WGP) [5-9].

Comprehensive data on the prevalence and distribution of HPV genotypes are informative in the planning of HPV/CC screening tools and with the rollout of HPV vaccines [10]. The hrHPV genotype distribution in Denmark [11, 12] and worldwide is well described in WGP and globally HPV16, 18, 52, 31, and 58 predominate $[10,13,14]$. Most studies suggest a different HPV genotype distribution in WLWH with increased frequency of non-16/18 HPV genotypes, which are not targeted by the 2 -and 4-valent HPV vaccines $[8,15,16]$.

Though, immune reconstitution induced by highly active antiretroviral therapy (HAART) might decrease the prevalence of HPV and cervical dysplasia, the effect of improved immunosurveillance remains controversial $[6,7]$. The increased longevity gained from HAART [17] may increase risk of exposure to HPV and provide the time required for progression to cancer.

Compared with other Western countries WGP in Denmark have high incidences of HPV and CC [11, 18, 19]. However, the prevalence and distribution of cervical HPV and dysplasia are unknown in WLWH in Denmark. Denmark offers a unique setting for studies on HPV and cervical dysplasia because of the well described HPV genotype distribution in WGP in Denmark [11, 19] and the possibility for linkage to nationwide registries on HIV and cytology results.

The aim of the present study was to assess the prevalence and distribution of cervical hrHPV and cytological abnormalities in WLWH in Denmark. Further, we aimed at identifying predictors of hrHPV and cytological abnormalities in WLWH in a setting with free access to CC screening, healthcare and HAART.

\section{Methods}

\section{Setting}

Denmark has a population of 5.6 million [20] and an estimated HIV prevalence among adults of $0.1 \%$ [21] - onefourth of these being women [22]. Medical care, including HAART, is tax-paid and provided free-of-charge to all people living with HIV (PLHIV). Treatment of HIV is restricted to eight specialized centres, of which six (treating $97 \%$ of Danish PLHIV) participated in the Study on HIV, cervical Abnormalities and infections in women in Denmark (SHADE) [23] (see below).

\section{Cervical screening in Denmark}

During the study period, The Danish National Board of Health recommended that women aged 23-49 years received cervical cytological testing every three years and women aged 50-65 years every five years [24]. In HIV guidelines cervical cytology is recommended twice the first year after HIV diagnosis and annually thereafter [25].

\section{The SHADE cohort}

The SHADE cohort is a multicentre, prospective, observational cohort study of WLWH in Denmark. Study procedures have been described previously [23]. In brief, study participants were consecutively enrolled during their outpatient visits from 1 February 2011 to 1 February 2012. Inclusion criteria were HIV-1 infection and $\geq 18$ years of age. Exclusion criteria were prior hysterectomy, pregnancy, alcohol and/or drug abuse impeding adherence to the protocol.

\section{Interview survey}

At entry, an interview including tobacco use, age at sexual debut, lifetime sexual partners, prior condyloma, HPV vaccination status, and contraceptive use etc. was performed. The EpiData Entry program was used for double manual data entry [26].

\section{Registries \\ Civil Registration System (CRS)}

The CRS is a national registry of all Danish residents [27]. A 10-digit personal identification number (PIN) is assigned to each individual at birth or immigration. The PIN was used to link the SHADE cohort, the Danish HIV Cohort Study (DHCS) and the The Danish Pathology Data Bank (DPDB).

\section{Danish HIV cohort study}

The DHCS is a prospective, observational, nationwide, multicentre cohort study of all PLHIV seen at the Danish HIV clinics since 1 January 1995. The cohort has been described in detail elsewhere [22].

\section{The Danish Pathology Data Bank (DPDB)}

The DPDB contains nationwide records of all pathology specimens [28]. Cytology samples prior to inclusion were retrieved to assess screening history and cytology results using the Systemized Nomenclature of Medicine (SNOMED) code of cervix uteri: T8x2\%, T8x3* and T83*.

\section{Data from the general population (the Horizon Study)}

Anonymised data on HPV (from the CLART assay) and cytology results from WGP included in the Danish Horizon Study, Copenhagen, Denmark, were retrieved from the authors [11]. 


\section{Cytology}

Cytological evaluation of SurePath samples was undertaken at Department of Pathology, Hvidovre, Copenhagen University Hospital (HVH). The outcomes were reported using the Bethesda 2001 system [29] and classified as normal, atypical cells of undetermined significance (ASCUS), low-grade squamous intraepithelial lesions (LSIL), or high-grade squamous intraepithelial lesions (HSIL) including atypical squamous cells - cannot exclude HSIL (ASC$\mathrm{H})$, atypical glandular cells (AGC) and adenocarcinoma in situ (AIS), and finally squamous cell-and adenocarcinoma.

\section{HPV DNA testing}

Cervical samples were examined by the CLART HPV2 assay (Genomica, Madrid, Spain) at Department of Pathology, HVH. PCR amplification of genotype specific HPV L1 fragments from 35 individual HPV genotypes was performed including $13 \mathrm{hrHPV}$ : HPV16, 18, 31, 33, $35,39,45,51,52,56,58,59$, and 68 [11]. Samples with invalid outcomes were retested. The second result was considered definitive [11].

\section{Statistical analysis}

Continuous variables were summarized as median and interquartile ranges (IQR) or mean and ranges and compared using the Wilcoxon rank sum test. Categorical variables were reported as counts and percentages and compared using the chi-square test or Fisher's exact test.

For comparison of HPV status, genotype distribution and cytology results study participants were matched 1:5 to WGP on prior screening history in accordance with the algorithm used in the Horizon study [11] and age with a tolerance of 2 years (choosing the 5 WGP numerically closest to the participant's age).

Univariate and multiple logistic regression analyses were used for identifying predictors of hrHPV expressed as odds ratios (OR) and $95 \%$ confidence intervals (CI). Nine candidate predictor variables were chosen a priori due to current knowledge on risk factors of HPV [6, 30, 31]; age $(18-29,30-50$ and $>50$ years of age), race, age at sexual debut $(<16$ versus $\geq 16$ years of age), HAART duration (years on HAART), AIDS prior to inclusion, smoking status, number of lifetime sexual partners $(<5$ versus $\geq 5$ ), use of hormonal contraceptives, and CD4 count at inclusion $(<200, \geq 200-349$ and $\geq 350$ cells $/ \mu \mathrm{L}$ ).

Predictors of ASCUS or worse (ASCUS+) were estimated by including the aforementioned variables, presence of cervical hrHPV and adherence to the general population CC screening program in the analysis. A subgroup analysis was performed in WLWH with ASCUS+ and cervical hrHPV only.

Duration of HAART, AIDS prior to inclusion and CD4 count are dependent covariates and where calculated using two models: A model where all variables but CD4 at inclusion was included and a model where duration of HAART and AIDS prior to inclusion were replaced by CD4. We only presented the OR of the CD4 count from the second model.

To account for multiple testing when comparing the distribution of genotypes, we applied Bonferoni correction. Therefore, in analyses of the $13 \mathrm{hrHPV}$ genotypes $p$-values smaller than 0.05:13 $(p<0.0038)$ were considered statistically significant. In the remaining analyses $p$-values $<0.05$ were considered statistically significant. For category variables with more than two outcome categories $(\mathrm{df}>1)$, we controlled for repeated testing by estimating the combined $p$-value. Individuals with missing explanatory values were excluded from the multiple regression analyses. The validity of the model was tested using the Hosmer and Lemeshow Goodness-of-Fit Test.

SAS statistical software version 9.3 (SAS Institute Inc., Cary, NC, USA) was used for data analysis. The matching of study participants and the HPV genotype distribution figures were performed in R 3.2.0 [32].

\section{Results}

\section{Characteristics of the cohort}

A total of 334 of the 1392 eligible WLWH in Denmark (in the DHCS) were included. At inclusion, median age and duration of HIV were 42.5 (IQR 36.8-48.3) and 11.3 (IQR 5.9-16.9) years (Table 1). Compared with WLWH from the DHCS not included, WLWH in SHADE were more likely to be sexually infected with HIV $(p=0.0015)$, have higher CD4 counts $(p=0.012)$, and increased probabilities of being on HAART with a suppressed viral load $(p=0.0042)$. Moreover, they had a higher uptake of both the annual HIV-and general population CC screening program $(p<0.0001$ and $p<0.0001)$ and a higher probability of the latest cytology result being normal $(p<0.0001)$ (Table 1).

\section{HPV prevalence}

Of 334 participants, 326 (97.6 \%) had a cervical swab performed. Of these 295 (90.5 \%) yielded sufficient DNA for analysis and were matched at 1:5 with 1475 WGP from the Horizon study (Fig. 1).

Overall HPV prevalence was higher in WLWH versus WGP $(26.4 \%$ versus $16.6 \%, p<0.0001)$ (Table 2$)$. Further, WLWH had a higher number of multiple infections with more genotypes diagnosed per sample $(p=0.030$ and $p=0.047$ ) (Table 2). Median age of WGP was due to the age matching criteria close to that of WLWH (stated above); 43.0 (IQR 37.0-49.0) years. The hrHPV prevalence according to age is shown in Fig. 2.

More WGP would have been protected by the 4-valent HPV vaccine than WLWH $(p=0.030)$, whereas both groups of women would receive a similar degree of 
Table 1 Characteristics of included and not included women living with HIV (WLWH) in Denmark from the Danish HIV Cohort Study (DHCS)

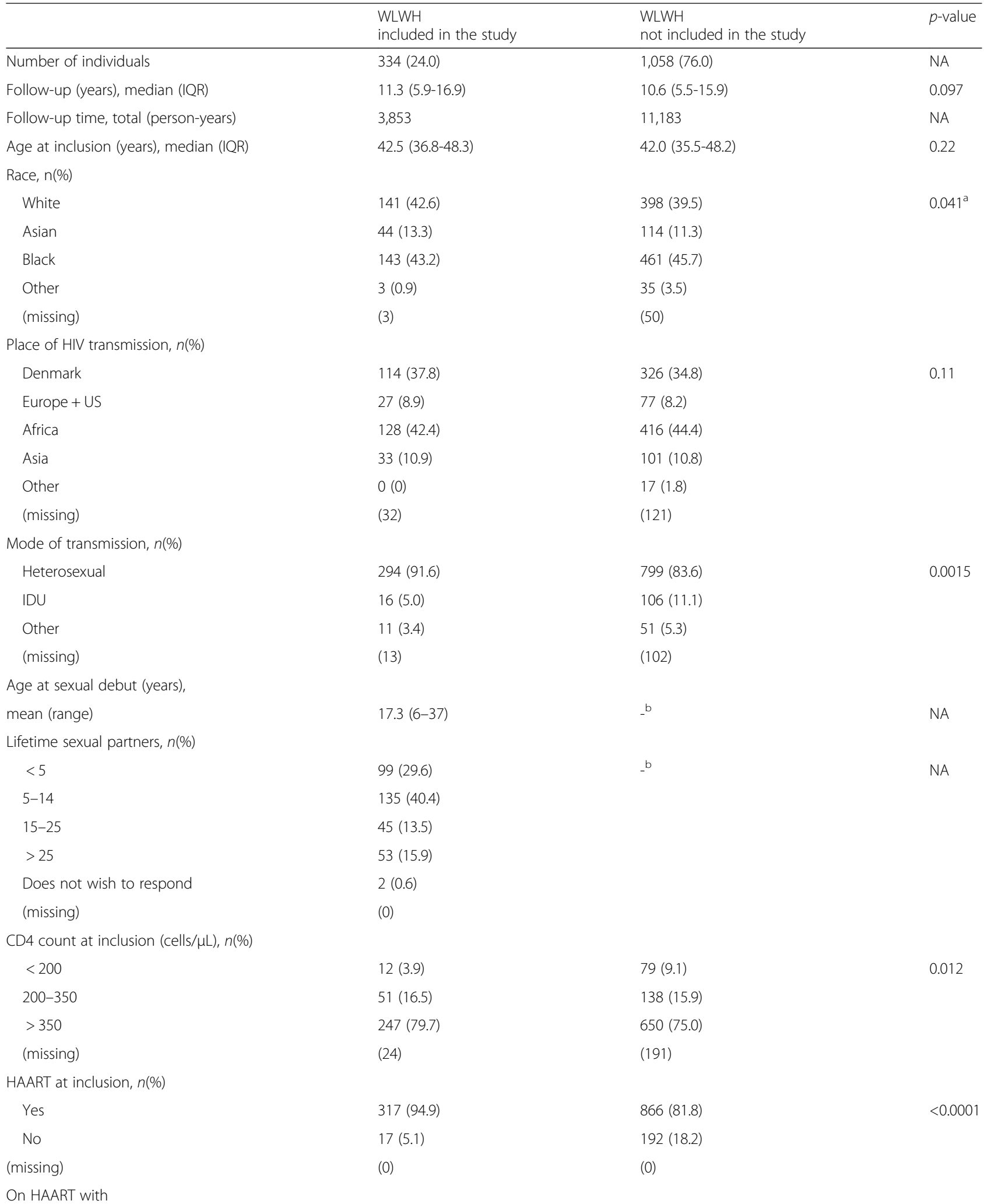


Table 1 Characteristics of included and not included women living with HIV (WLWH) in Denmark from the Danish HIV Cohort Study (DHCS) (Continued)

\begin{tabular}{|c|c|c|c|}
\hline \multicolumn{4}{|l|}{ HIV RNA < 40 copies/mL, $n(\%)$} \\
\hline Yes & $250(83.6)$ & $576(75.5)$ & \multirow[t]{3}{*}{0.0042} \\
\hline No & $49(16.4)$ & $187(24.5)$ & \\
\hline (missing) & $(18)$ & (103) & \\
\hline \multicolumn{4}{|c|}{ Cervical cytology within the past 1 year, $n(\%)^{c}$} \\
\hline Yes & $124(37.1)$ & $225(21.3)$ & \multirow[t]{3}{*}{$<0.0001$} \\
\hline No & $210(62.9)$ & $833(78.7)$ & \\
\hline (missing) & $(0)$ & (0) & \\
\hline \multicolumn{4}{|c|}{ Cervical cytology within the past $3 / 5$ years - depending on age for women age $23-65$ years, $n(\%)^{d}$} \\
\hline Yes & $227(67.7)$ & $429(40.6)$ & \multirow[t]{4}{*}{$<0.0001$} \\
\hline No & $96(28.7)$ & $555(52.5)$ & \\
\hline Outside target age group & $11(3.3)$ & $74(7.0)$ & \\
\hline (missing) & $(0)$ & (0) & \\
\hline \multicolumn{4}{|l|}{ Last cytology result, $n(\%)$} \\
\hline Normal & $242(72.5)$ & $513(48.5)$ & \multirow[t]{3}{*}{$<0.0001$} \\
\hline Abnormal & $9(2.7)$ & $21(2.0)$ & \\
\hline No prior cytology obtained & $83(24.9)$ & $524(49.5)$ & \\
\hline \multicolumn{4}{|l|}{ HPV vaccination prior to inclusion, $n(\%)$} \\
\hline Yes (4-valent HPV vaccine) & $4(1.2)$ & $-\mathrm{b}$ & \multirow[t]{5}{*}{ NA } \\
\hline Yes (2-valent HPV vaccine) & $0(0)$ & & \\
\hline Yes (do not know name of vaccine) & $1(0.3)$ & & \\
\hline No & $329(98.5)$ & & \\
\hline (missing) & $(0)$ & & \\
\hline
\end{tabular}

IDU intravenous drug user, NA not applicable, HAART Highly active antiretroviral therapy, HPV Human papillomavirus

a There was no difference in distribution of race between groups if the category "other" was removed from the "Race" variable ( $p=0.45)$, ${ }^{b}$ No information available, ${ }^{\mathrm{C}} \mathrm{As}$ recommended in women living with HIV (we studied the past year + a 3-month grace period), ${ }^{\mathrm{d}}$ As recommended in the general population, where women aged 23-49 years were invited for cervical cancer screening every third year and women aged 50-65 years every fifth year (we studied the past $3 / 5$ years + a 3-month grace period)

protection from the 9-valent HPV vaccine based upon their HPV genotype distribution $(p=0.14)$ (Table 2).

\section{HPV genotype distribution}

Figure 3 shows the prevalence of hrHPV genotypes with respect to race: i) overall, ii) in WLWH and WGP with normal cytological findings, and iii) WLWH and WGP with ASCUS+. Overall, the six most frequent high-risk genotypes in WLWH were HPV58 $(n=21,7.1 \%), 52$ $(n=16,5.4 \%), 16 \quad(n=14,4.8 \%), 51 \quad(n=12,4.1 \%)$, $18(n=10,3.4 \%)$ and $33(n=10,3.4 \%)$ versus $16(n=60$, $4.1 \%), 52(n=41,2.8 \%), 58(n=35,2.4 \%), 31 \quad(n=32$, $2.2 \%), 51(n=28,1.9 \%)$ and $33(n=26,1.8)$ in WGP (Fig. 3, Additional file 1: Table S1).

\section{HPV genotype distribution according to race}

There was no difference in prevalence of hrHPV in WLWH of White, Asian and Black race $(26.6 \%, 16.2 \%$ and 29.0, $(p=0.30))$. We compared genotype distribution in WLWH of different races and found that Black
WLWH had a higher risk of HPV58 and 56 compared with WGP $(p<0.0001$ and $p=0.0023)$ (Fig. 3$)$, while no significant differences in hrHPV genotype distribution were found between White and Asian WLWH compared with WGP (Fig. 3).

\section{HPV genotype distribution in women with normal cytology}

In women with normal cytology the hrHPV prevalence was $22.0 \%$ in WLWH versus $13.0 \%$ in WGP $(p=0.0004)$. HPV58 $(n=13,5.1 \%), 52(n=11,4.3 \%)$, $51(n=8,3.1 \%)$ versus HPV16 $(n=49,3.5 \%), 58(n=30$, $2.2 \%)$ and $52(n=30,2.2 \%)$ predominated in WLWH and WGP, respectively (Fig. 3, Additional file 2: Table S2).

\section{HPV genotype distribution in women presenting with ASCUS+}

In WLWH and WGP with ASCUS+ $61.8 \%$ versus $49.0 \%$ were hrHPV-positive $(p=0.25)$. 


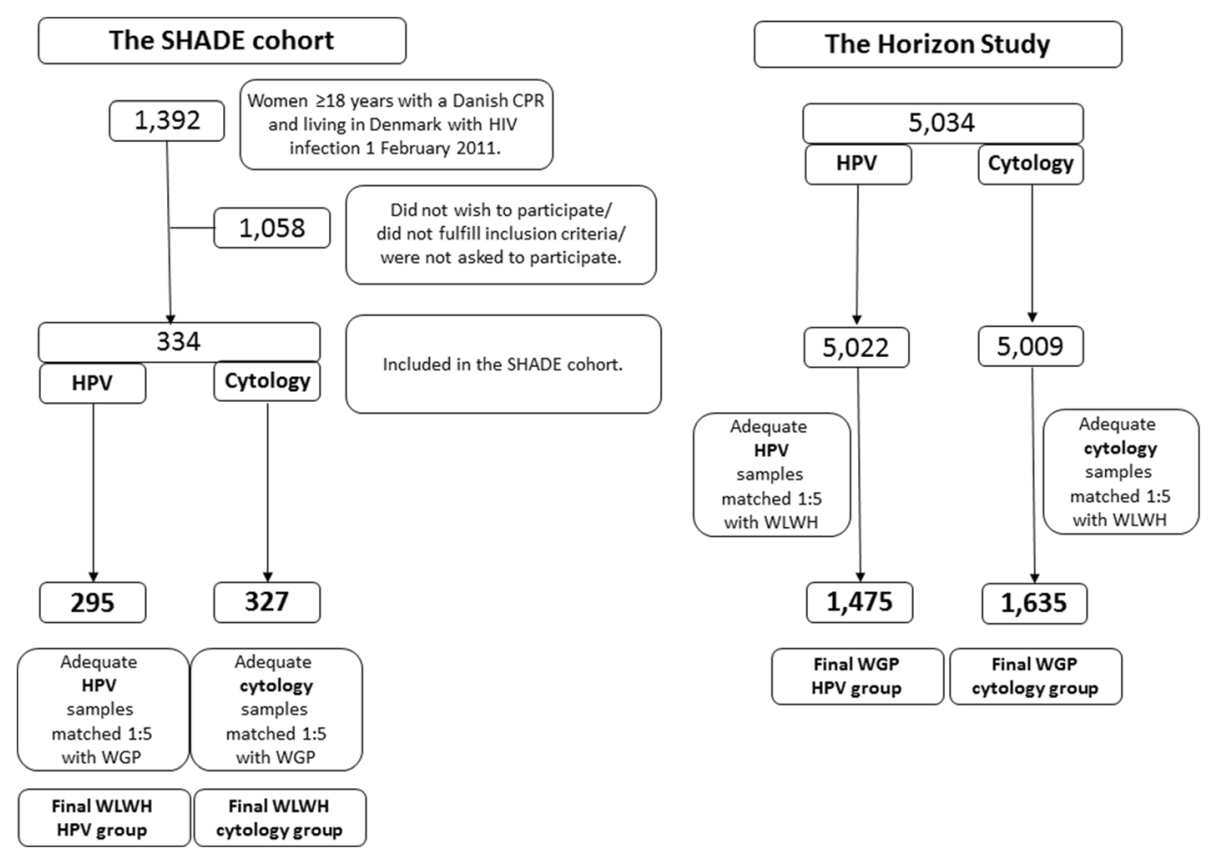

Fig. 1 Flowchart of women living with HIV (WLWH) from the SHADE cohort and women from the general population (WGP) from the Horizon study matched 1:5 on prior screening history and age

Table 2 Prevalence of high-risk human papillomavirus (HPV) in women with sufficient DNA for analyses in women living with HIV compared to women matched (1:5) on prior screening history and age from the general Danish population

\begin{tabular}{lll}
\hline & $\begin{array}{l}\text { Women living with HIV } \\
295\end{array}$ & $\begin{array}{l}\text { Women from the general population } \\
1,475\end{array}$ \\
\hline $\begin{array}{l}\text { High-risk HPV positive, } n(\%) \\
\text { Yes }\end{array}$ & $78(26.4)$ & $245(16.6)$ \\
No & $217(73.6)$ & $1,230(83.4)$ \\
Number of genotypes, mean (range) & $1.54(1-4)$ & $1.38(1-5)$ \\
Number of infections, $n(\%)$ & & $182(74.3)$ \\
Single & $48(61.5)$ & $63(25.7)$ \\
Multiple $(>1)$ & $30(38.5)$ & 0.0001 \\
All high-risk genotypes present targeted by the 4-valent HPV vaccine ${ }^{a}, n(\%$ of the HPV positive patients) \\
Yes & $8(10.3)$ & $52(21.2)$ \\
No & $70(89.7)$ & $193(78.8)$
\end{tabular}

All high-risk genotypes present targeted by the 9 -valent vaccine ${ }^{\mathrm{b}}, n(\%$ of the HPV positive patients)
Yes
$42(53.9)$
$155(63.3)$
No
$36(46.1)$
$90(36.7)$

Presence of $\geq 1$ high-risk genotypes targeted by the 4-valent vaccine ${ }^{a}, n(\%$ of the HPV positive patients)
Yes
21 (26.9)
76 (31.0)

No

57 (73.1)

$169(69.0)$

Presence of $\geq 1$ high-risk genotypes targeted by the 9 -valent vaccine ${ }^{\mathrm{b}}, \mathrm{n}(\%$ of the HPV positive patients)
Yes
61 (78.2)
194 (79.2)
No
17 (21.8)
51 (20.8)

a Targeting HPV6, HPV11, HPV16 and HPV18. HPV6 and HPV11 are low-risk genotypes and not included in this analysis

${ }^{\mathrm{b}}$ Targeting HPV6, HPV11, HPV16, HPV18, HPV31, HPV33, HPV45, HPV52, and HPV58. HPV6, and HPV11 are low-risk genotypes and not included in this analysis 


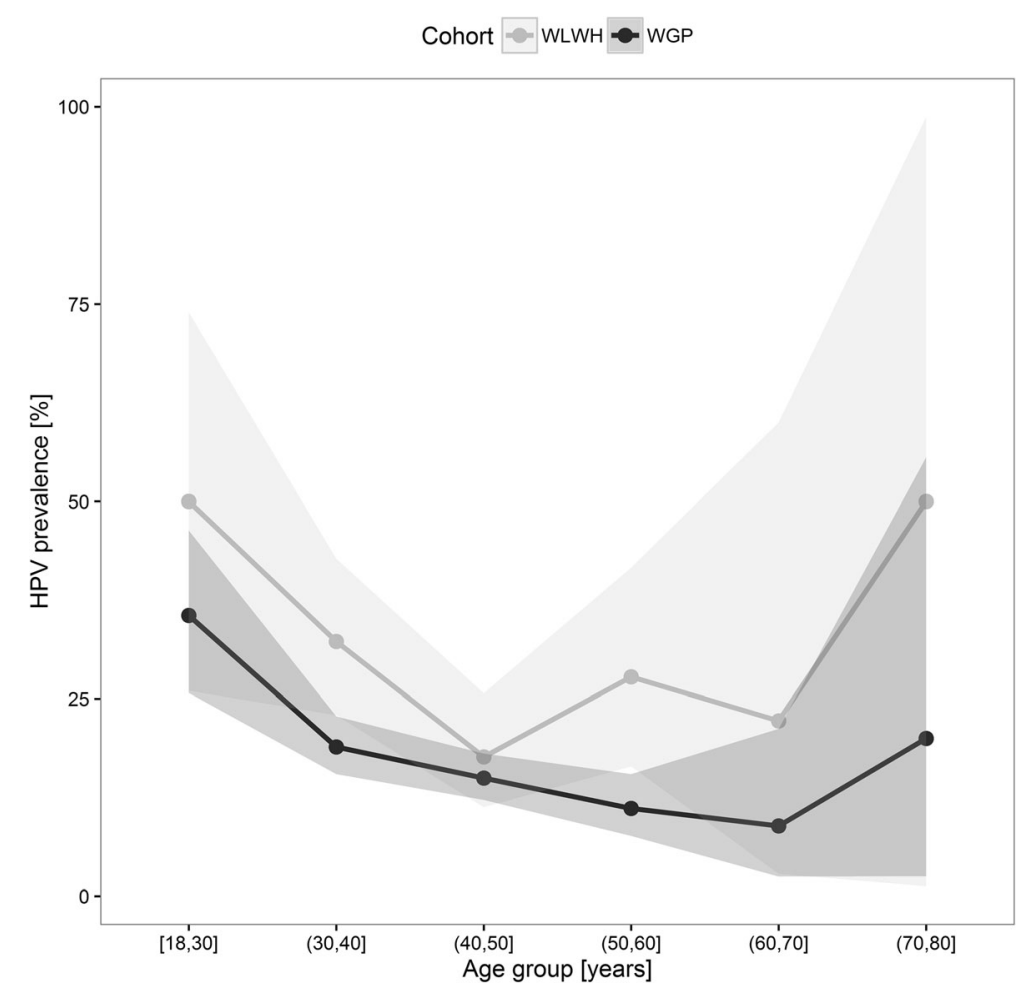

Fig. 2 Prevalence of high-risk human papillomavirus (HPV) according to age group in women living with HIV (WLWH) and women from the general population (WGP)

Distribution of genotypes in descending order in WLWH and WGP presenting with ASCUS+ were HPV58 $(n=8,23.5 \%), 16(n=7,20.6 \%)$ and $56(n=5,14.7 \%)$ versus HPV52 ( $n=11,14.1 \%), 16(n=10,12.8 \%)$ and 45 ( $n=9,11.5 \%)$ (Fig. 3, Additional file 3: Table S3).

\section{Predictors of HPV}

Short duration of HAART (adjusted OR per year 0.90 (95 \% CI 0.84-0.96)), AIDS prior to inclusion (adjusted OR 3.61 (95\% CI 1.75-7.46)), $\geq 5$ lifetime sexual partners (adjusted OR 2.20 (95\% CI 1.08-4.49)), sexual debut $<16$ years of age (adjusted OR 2.05 (95 \% CI 1.03-4.10)) and CD $4<350$ cells $/ \mu \mathrm{L}$ (adjusted OR 2.53 (95 \% CI 1.20-5.40)) predicted prevalent hrHPV (Table 3).

\section{Cervical cytological abnormalities}

Five $(1.5 \%)$ of the 332 cytology samples received from WLWH were inadequate for evaluation leaving 327 for interpretation. These were matched with cytology results from 1635 WGP (Fig. 1). ASCUS+ was prevalent in 34 $(10.4 \%)$ versus $85(5.2 \%)$ WLWH and WGP, $(p=0.0003)$. Cytological abnormalities in WLWH and WGP were distributed as follows: ASCUS: 8 (2.5\%) versus $42(2.6 \%)$, $(p=0.90)$; LSIL: 20 (6.1 \%) versus $23(1.4 \%),(p<0.0001)$ and HSIL: $6(1.8 \%)$ versus $20(1.2 \%),(p=0.38)$. No WLWH or WGP presented with carcinoma.

\section{Predictors of cytological abnormalities}

HrHPV predicted ASCUS+ (adjusted OR 6.91 (95 \% CI 2.91-16.42)) (Additional file 4: Table S4). However, in the subgroup of WLWH with hrHPV short duration of HAART predicted ASCUS+ (adjusted OR 0.83 (95\% CI 0.71-0.97)) (Additional file 5: Table S5).

In all adjusted analyses we checked the effect of missing values on outcome by adding an extra category with missing values. This had no impact on the estimates.

\section{Discussion}

In this multicentre, cross-sectional cohort study of WLWH in Denmark, we found a higher prevalence of cervical hrHPV in WLWH compared with WGP matched on prior screening history and age. Further, WLWH had a higher number of hrHPV genotypes and more carried multiple hrHPV infections. Presence of $\geq 1$ genotypes covered by the 9-valent HPV vaccine was higher in WLWH and a higher number of WGP had all present genotypes covered by the 4-valent HPV vaccine. WLWH had a different distribution of hrHPV genotypes and this difference was mainly attributed to WLWH of Black race. There was a higher risk of ASCUS+ in WLWH, due to a higher prevalence of LSIL. Finally, cervical HPV and ASCUS+ were predicted by short duration of HAART. 


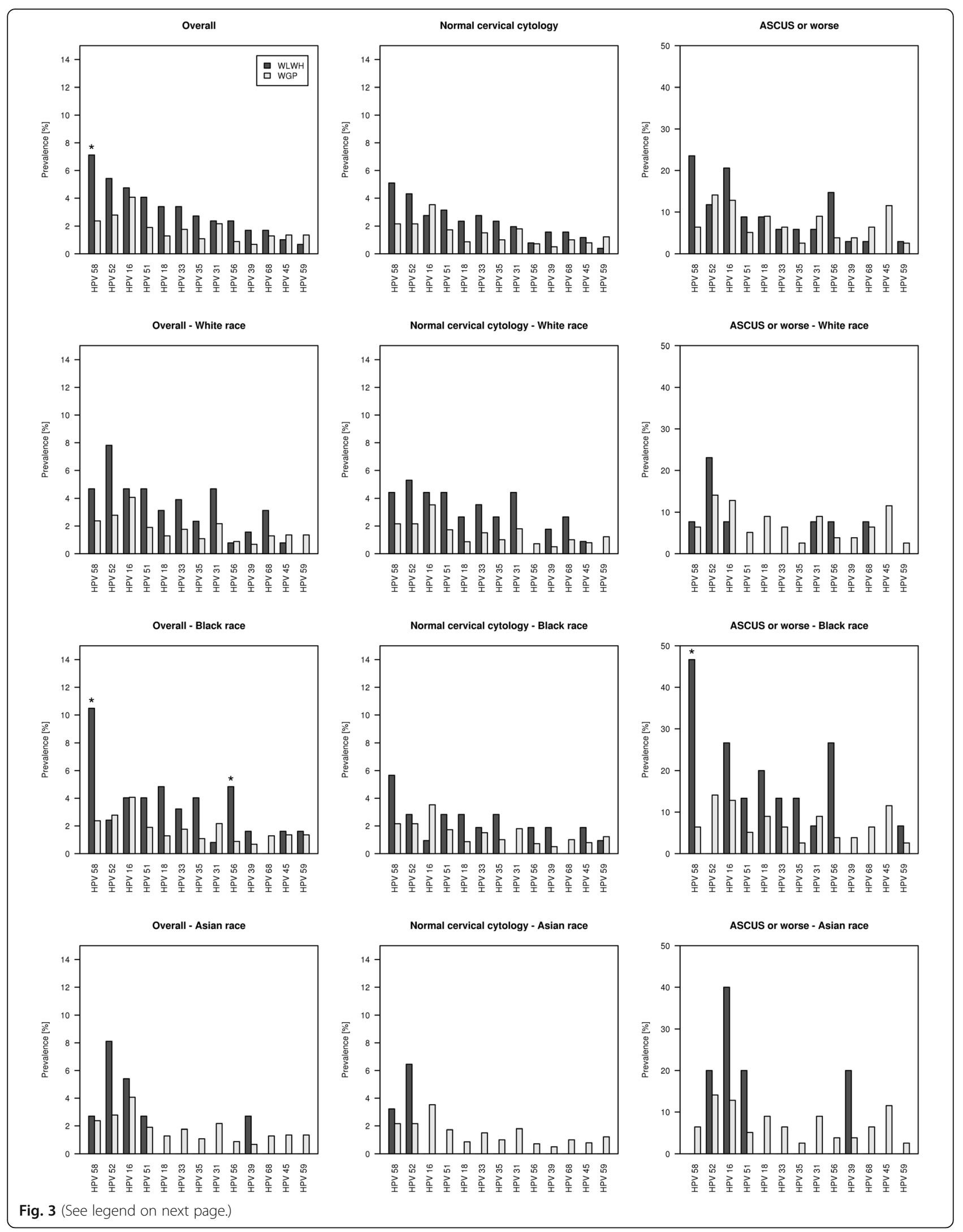


(See figure on previous page.)

Fig. 3 Cervical high-risk human papillomavirus (HPV) genotype distribution in women living with HIV (WLWH) compared to women from the general population (WGP); i) overall, ii) in women with normal cervical cytology, and iii) women with atypical cells of undetermined significance (ASCUS) or worse. Distribution is presented i) overall, ii) comparing WGP to WLWH of White race, iii) comparing WGP to WLWH of Black race and iv) comparing WGP to WLWH of Asian race. Please notice the different scale on the y-axis in the "ASCUS or worse" plots

\section{HPV prevalence}

Prevalent HPV infection is dependent on age with a peak prevalence in women in the early 20 s followed by a steady decline and a second, but smaller peak in women $\geq 45$ years of age attributed to either new acquisition or viral persistence $[2,14]$. Therefore, we chose to match WLWH and WGP on age and prior cytology/histology results. The overall hrHPV prevalence in WLWH of $26.4 \%$ was lower than the hrHPV prevalence found in the European MACH-1 collaborative group of WLWH with a prevalence close to $50 \%$ [15], however participants in the MACH study were younger (median age: 35 versus 42.5 years), less likely to be on HAART (69.7\% versus $94.9 \%$ ) and a different assay (Hybrid Capture II) was used for HPV detection. On a global scale the highest prevalence of HPV in WGP with normal cytological findings is found in Africa (24\%) [14]. Though, the overall hrHPV prevalence was highest in WLWH of Black race, this difference was not significant.

\section{HPV genotype distribution}

Studies of WGP across all continents have repeatedly identified hrHPV16 and 18 - accounting for about $70 \%$ of all CCs $[2,6]$ - to be among the most prevalent [8]. In agreement with others we found HPV58 and 52 to be prevailing in WLWH $[8,16]$. Though, the numbers were small, this higher risk of HPV58 was mainly carried by women of Black race, as no significant difference in genotype distribution, was detected when comparing WGP with WLWH of White and Asian race. While the 2-and 4-valent HPV vaccines are anticipated to reduce the burden of HPV-related cancers in WGP [13], the impact of these HPV vaccines in WLWH is less clear. More than $20 \%$ of WGP had all genotypes present covered by the 4-valent HPV vaccine, whereas only one-tenth of genotypes were accounted for amongst WLWH. The novel 9-valent HPV-vaccine targeting HPV6, 11, 16, 18, 31, 33, 45, 52, and 58 has the potential to prevent about $90 \%$ of CC cases in WGP if administered before sexual onset [33] might be better suited for the HIV population.

\section{Predictors of HPV}

The molecular mechanisms leading to the increased risk of HPV in PLHIV are poorly understood [34]. Tugizov et al. suggested that HIV-proteins enable initial HPV infection by disrupting the epithelial tight junctions [34]. Moreover, immune defects associated with HIV infection probably contribute to the HPV pathogenesis by preventing spontaneous clearance of HPV [6]. A recent study found that in WLWH every month on HAART reduced the detection risk of any cervical HPV infection by $9 \%$ [30]. Likewise, short duration of HAART predicted HPV in the current study. Moreover, sexual behavior such as early sexual debut and $\geq 5$ lifetime sexual partners predicted prevalent HPV and so did variables associated with a compromised immune system such as CD $4<350$ cells $/ \mu \mathrm{L}$ and AIDS prior to inclusion.

\section{Cytological abnormalities}

Overall, WLWH had more cytological abnormalities, due to a higher prevalence of LSIL. A higher prevalence of LSIL, but not ASCUS and HSIL, in WLWH is also reported by others [35]. Most LSILs are transient and resolve within 1-2 years [36], and are most often not treated according to current Danish guidelines. A high proportion of WLWH included and not included in the study; $28.7 \%$ and $52.5 \%$, had not been screened for CC in the preceding 3 to 5 years. We have previously assessed low screening attendance in this cohort of WLWH and support the idea of cytology as part of an annual medical HIV review, integration of HIV care and cervical screening in a single clinic setting and targeted public health messages aimed at health care professionals at HIV centres, general practitioners and WLWH [37]. However, the low screening attendance does not explain differences in cytological abnormalities between WLWH and WGP, since women were matched on prior screening history.

\section{Predictors of cervical dysplasia}

Predictors of cervical dysplasia and CC are those also associated with HPV infection such as smoking, early sexual debut, number of lifetime sexual partners, hormonal contraceptives and STDs other than HPV [6, 7]. We have earlier reported that only a very few of WLWH in SHADE presented with STDs other than HPV [23] and therefore this variable was not adjusted for. Not surprisingly, hrHPV predicted ASCUS+. Since hrHPV causes most cases of dysplasia and basically all cases of CC [6], this variable could blur the effect of other covariates. We therefore performed a sensitivity analysis in WLWH with hrHPV, and in consistence with our findings regarding HPV we found that short duration of HAART predicted ASCUS+. 
Table 3 Unadjusted and adjusted odds ratios for predictors of cervical high-risk human papillomavirus (HPV) infection in women living with HIV with sufficient DNA for HPV analysis $(n=295)$

\begin{tabular}{|c|c|c|c|c|c|c|}
\hline Predictors of $\mathrm{HPV}^{\mathrm{a}}$ & $\begin{array}{l}\text { HPV-positive group } \\
(n=78)\end{array}$ & $\begin{array}{l}\text { HPV-negative group } \\
(n=151)\end{array}$ & Unadjusted odds ratios & $p$-value & Adjusted odds ratios ${ }^{b}$ & $p$-value \\
\hline \multicolumn{7}{|c|}{ Age at 1 February 2011 (inclusion), $n(\%)$} \\
\hline $18-29$ years & $9(11.5)$ & $9(4.2)$ & 1.00 & - & 1.00 & - \\
\hline $30-50$ years & $51(65.4)$ & $161(74.2)$ & $0.32(0.12-0.84)$ & 0.02 & $0.43(0.14-1.33)$ & 0.14 \\
\hline$>50$ years & $18(23.1)$ & $47(21.7)$ & $0.38(0.13-1.12)$ & 0.08 & $0.51(0.13-1.92)$ & 0.32 \\
\hline (missing) & $0(0)$ & (0) & & & & \\
\hline Combined p-value & & & & 0.068 & & 0.32 \\
\hline \multicolumn{7}{|l|}{ Race, $n(\%)$} \\
\hline White & $34(44.7)$ & $94(44.1)$ & 1.00 & - & 1.00 & - \\
\hline Asian & $6(7.9)$ & $31(14.6)$ & $0.54(0.21-1.40)$ & 0.20 & $0.67(0.23-1.92)$ & 0.46 \\
\hline Black & $36(47.4)$ & $88(41.3)$ & $1.13(0.65-1.96)$ & 0.66 & $0.38(0.67-2.87)$ & 0.38 \\
\hline (missing) & (2) & (4) & - & - & & \\
\hline Combined $p$-value & & & & 0.31 & & 0.35 \\
\hline \multicolumn{7}{|l|}{ Sexual debut, $n(\%)$} \\
\hline$\geq 16$ years of age & $51(65.4)$ & $160(73.7)$ & 1.00 & - & 1.00 & - \\
\hline$<16$ years of age & $27(34.6)$ & $57(26.3)$ & $1.49(0.85-2.59)$ & 0.16 & $2.05(1.03-4.10)$ & 0.042 \\
\hline (missing) & (0) & (0) & - & & & \\
\hline \multicolumn{7}{|l|}{ HAART duration, (years) } \\
\hline Median (IQR) & $4.6(2.0-10.4)$ & $8.8(4.6-12.2)$ & $0.91(0.86-0.96)$ & 0.0013 & $0.90(0.84-0.96)$ & 0.0011 \\
\hline (missing) & (2) & (9) & & & & \\
\hline \multicolumn{7}{|l|}{ AIDS prior to inclusion, $n(\%)$} \\
\hline No & $55(71.4)$ & $191(88.4)$ & 1.00 & - & 1.00 & - \\
\hline Yes & $22(28.6)$ & $25(11.6)$ & $0.33(0.17-0.63)$ & 0.0007 & $3.61(1.75-7.46)$ & 0.0005 \\
\hline (missing) & (1) & (1) & & & & \\
\hline \multicolumn{7}{|l|}{ Smoking status, $n(\%)$} \\
\hline Current smoker/Ex-smoker & $33(42.3)$ & $92(42.4)$ & 1.00 & - & 1.00 & - \\
\hline Never smoker & $45(57.7)$ & $125(57.6)$ & $1.00(0.59-1.69)$ & 0.99 & $1.31(0.65-2.63)$ & 0.45 \\
\hline (missing) & (0) & (0) & & & & \\
\hline \multicolumn{7}{|c|}{ Number of lifetime sexual partners at inclusion, $n(\%)$} \\
\hline$<5$ & $36(25.0)$ & $54(36.0)$ & 1.00 & - & 1.00 & - \\
\hline$\geq 5$ & $108(75.0)$ & $96(64.0)$ & $1.83(1.00-3.36)$ & 0.05 & $2.20(1.08-4.49)$ & 0.03 \\
\hline (missing) & (0) & (1) & & & & \\
\hline \multicolumn{7}{|l|}{ Use of hormonal contraceptives, } \\
\hline$n(\%)$ & $12(8.3)$ & $9(6.0)$ & 1.00 & - & 1.00 & - \\
\hline Yes & $132(91.7)$ & $142(94.0)$ & $0.89(0.33-2.38)$ & 0.82 & $1.29(0.40-4.10)$ & 0.67 \\
\hline No & (0) & (0) & & & & \\
\hline \multicolumn{7}{|l|}{ (missing) } \\
\hline \multicolumn{7}{|c|}{ CD4 count at inclusion (cells $/ \mu \mathrm{L}$ ), } \\
\hline$>350$ & $45(69.2)$ & $173(83.2)$ & 1.00 & - & 1.00 & - \\
\hline 200-350 & $15(23.1)$ & $28(13.5)$ & $2.06(1.02-4.18)$ & 0.045 & $2.53(1.20-5.40)$ & 0.015 \\
\hline$<200$ & $5(7.7)$ & $7(3.4)$ & $2.75(0.83-9.06)$ & 0.10 & $2.70(0.78-9.33)$ & 0.12 \\
\hline (missing) & (13) & (9) & & & & \\
\hline Combined $p$-value & & & & 0.0496 & & 0.023 \\
\hline
\end{tabular}

HAART $=$ Highly active antiretroviral therapy

${ }^{a}$ Two models are shown in the table: Age, race, sexual debut, smoking status, number of lifetime sexual partners and use of hormonal contraceptives were included in both models, whereas HAART duration and AIDS prior to inclusion were included in the first model and replaced by CD4 at inclusion inthe second model, We only presented the ORs of the CD4 count from the second model, ${ }^{\text {'T }}$ The validity of the model was tested using the Hosmer and Lemeshow Goodness-of-Fit Test 


\section{Strengths and limitations}

We have a very well-described cohort due to the DHCS and DPDB and were able to match on prior cytological outcomes. Compared to other Western countries Danish women represent a CC high-risk population [18], therefore comparison to WGP is essential in interpretation of results.

Possible limitations are that WLWH included in SHADE were more likely to comply with CC screening programs and to have a previous normal cytology result. Therefore estimates regarding cytological abnormalities in WLWH might be conservative. The effect of HAART duration can be confounded by other time-scales such as duration of HIV. Moreover, racial origin of the WGP group was not available for direct comparison to the SHADE cohort. Furthermore, a higher number of inadequate HPV samples were collected in the present study compared to ordinary CC screening samples included in the Horizon study, probably as a consequence of sampling by personnel less trained in gynecological routines. Finally, this is a cross-sectional study giving information on HPV infection at the time of one single sampling. The limitation in this design is that without previous or subsequent sampling results, any HPV infection observed may be a transient infection.

\section{Conclusions}

WLWH had a higher risk of being cervical hrHPV positive, a higher frequency of multiple infections, a different genotype distribution and more cytological abnormalities than WGP. Cervical HPV and ASCUS+ were predicted by short duration of HAART.

\section{Additional files}

Additional file 1: Table S1. Comparison of the overall prevalence of high-risk HPV genotypes in women living with HIV (WLWH) and age-matched women from the general population (WGP). (DOCX $20 \mathrm{~kb}$ )

Additional file 2: Table S2. Comparison of the prevalence of high-risk HPV genotypes in women living with HIV (WLWH) and women from the general population (WGP) with normal cytological findings. (DOCX $20 \mathrm{~kb}$ )

Additional file 3: Table S3. Comparison of the prevalence of high-risk HPV genotypes in women living with HIV (WLWH) and women from the general population (WGP) with abnormal cytological findings (ASCUS or worse). (DOCX $20 \mathrm{~kb}$ )

Additional file 4: Table S4. Unadjusted and adjusted odds ratios for predictors of ASCUS or worse (ASCUS+) in women living with HIV with adequate cytology samples. (DOCX $27 \mathrm{~kb}$ )

Additional file 5: Table S5. Unadjusted and adjusted odds ratios for predictors of ASCUS or worse in women living with HIV with adequate cytology samples and positive for cervical high-risk human papillomavirus. (DOCX $26 \mathrm{~kb}$ )

\section{Abbreviations}

CC: Cervical cancer; Cl: Confidence interval; CRS: Civil Registration System; DF: Degrees of freedom; DHCS: Danish HIV Cohort Study; DPDB: The Danish Pathology Data Bank; HAART: Highly active antiretroviral therapy;

HPV: Human papillomavirus; HVH: Hvidovre, Copenhagen University Hospital;
MSM: Men who have sex with men; OR: Odds ratio; PIN: Personal identification number; PLHIV: People living with HIV; SHADE: Study on HIV, cervical Abnormalities and infections in women in Denmark; STD: Sexually transmitted diseases; WGP: Women from the general population; WLWH: Women living with HIV

\section{Acknowledgements}

We are first and foremost grateful to all the women living with HIV for participating in the study. We further thank the staff of our clinical departments for their continuous support and enthusiasm at the participating centres in the SHADE cohort: Department of Infectious Diseases, Hvidovre, Copenhagen University Hospital (AM Lebech, K Thorsteinsson); Department of Infectious Diseases, Copenhagen University Hospitals, Rigshospitalet (TL Katzenstein, FF Rönsholt); Department of Infectious Diseases, Odense University Hospital (IS Johansen); Department of Infectious Diseases, Aarhus University Hospitals, Skejby (M Storgaard); Department of Infectious Diseases, Aalborg University Hospital (G Pedersen); Department of Infectious Diseases, Hillerød Hospital (LN Nielsen). Moreover, we are grateful to the study group behind the Horizon Study for providing us with data on women from the general population.

\section{Funding}

This work was supported by the Danish Cancer Society and the AIDS Foundation; the Aragon Foundation; the Foundation of Aase and Ejnar Danielsens and the Foundation of Anna and Preben Simonsen. Grant numbers do not apply in a Danish setting. The sponsors of the study had no role in study design, data collection, data analysis, data interpretation or writing of the article.

\section{Availability of data and materials}

We believe that all data of relevance for the present study is available within the manuscript and in the Additional file 1: Table S1, Additional file 2: Table S2, Additional file 3: Table S3, Additional file 4: Table S4 and Additional file 5: Table S5. We are open to collaboration and in involving other researchers in our work. However, we strongly feel that we cannot make a full dataset publicly available for the following reasons: We are extremely concerned about confidentiality - since these patients may be identified by combinations of person-specific characteristics within the database, and the database includes sensitive data such as HIV status, previous diagnosis of AIDS, data on intravenous drug use and sexual preferences. Further we are concerned that public access to our dataset would compromise our ability to publish future articles on WLWH included in the SHADE.

\section{Authors' contributions}

KT contributed to conception and design of the study, included patients, performed interviews and gynaecological examinations, analysed and interpreted data, and drafted the manuscript. SL, biostatistician, was involved in analysis and interpretation of data, and critically reviewed the manuscript. MS contributed to conception and design of the study, included patients, performed interviews and gynaecological examinations, and critically reviewed the manuscript. TLK contributed to conception and design of the study, included patients, performed interviews and gynaecological examinations, and critically reviewed the manuscript. FR included patients, performed interviews and gynaecological examinations, and critically reviewed the manuscript. ISJ contributed to conception and design of the study, included patients, performed interviews and gynaecological examinations, and critically reviewed the manuscript. GP included patients, performed interviews, and critically reviewed the manuscript. LH, performed gynaecological examinations and critically reviewed the manuscript. LNN included patients and performed interviews, and critically reviewed the manuscript. LN, performed gynaecological examinations in WLWH needing follow-up and provided valuable information on gynaecological procedures in Denmark, and critically reviewed the manuscript. JB was in charge of the analyses of HPV, and critically reviewed the manuscript. NO, head of the DHCS, critically reviewed the manuscript. AML, principal investigator, contributed to conception and design of the study, included patients and performed interviews and gynaecological examinations, was involved in analysis and interpretation of data, and critically reviewed the manuscript. All authors read and approved the final manuscript. 


\section{Competing interests}

$\mathrm{KT}$ has received research funding from Abbott and honoraria from JanssenCilag, BMS and GlaxoSmithKline/Niiv. JB has in the past served as paid advisor or advisory board member to Roche Molecular Systems, Genomica SAU, and BD Diagnostics. He has received honoraria from Roche, Qiagen, Hologic, Genomica and Bd Diagnostics for lectures. HW has received research funding and honoraria from Roche Molecular, Hologic, and Novo Nordic. TLK has received research funding and/or honoraria from Bristol-Myers Squibb, Merck Sharp \& Dohme, GlaxoSmithKline/Viiv, Abbott, Gilead, and Janssen-Cilag. AML has received research funding from Abbott and honoraria from Bristol-Myers Squibb, Merck Sharp \& Dohme, GlaxoSmithKline, Boehringer Ingelheim and Janssen-Cilag. SL, MS, $F R, I J, L H, L N N, L N$ and $N O$ report no conflicts of interest.

\section{Consent to publish}

Not applicable.

\section{Ethics approval and consent to participate}

At entry, written and oral informed consent was obtained from all participants. The study and the DHCS were approved by the Danish Data Protection Agency (2015-231-0126, 2012-58-0004 and 2012-41-0005). Further, the study was approved by the Danish Regional Committee on Health Research Ethics (approval numbers: H-3-2010-119 and H-2-2014-102).

\section{Meetings where the work has been presented}

Oral at the 15th European AIDS Conference in Barcelona, Spain, October 21-24, 2015. Oral at the $2^{\text {nd }}$ Nordic HIV \& Women Meeting, Copenhagen, December 9, 2015

\section{Author details}

'Department of Infectious Diseases, Hvidovre, Copenhagen University Hospital, Copenhagen, Denmark. ${ }^{2}$ Department of Infectious Diseases, Skejby, Aarhus University Hospital, Aarhus, Denmark. ${ }^{3}$ Department of Infectious Diseases, Copenhagen University Hospital, Rigshospitalet, Copenhagen, Denmark. ${ }^{4}$ Institute of Clinical Medicine, University of Copenhagen, Copenhagen, Denmark. ${ }^{5}$ Clinical Research Center, Hvidovre, Copenhagen University Hospital, Copenhagen, Denmark. ${ }^{6}$ Department of Infectious Diseases, Odense University Hospital, Odense, Denmark. ${ }^{7}$ Department of Infectious Diseases, Aalborg University Hospital, Aalborg, Denmark. ${ }^{8}$ Department of Obstetrics and Gynaecology, Aalborg University Hospital, Aalborg, Denmark. ${ }^{9}$ Department of Infectious Diseases, Nordsjællands Hospital, Hillerød, Denmark. ${ }^{10}$ Department of Obstetrics and Gynaecology, Hvidovre, Copenhagen University Hospital, Copenhagen, Denmark. ${ }^{11}$ Molecular Pathology Laboratory, Department of Pathology, Hvidovre, Copenhagen University Hospital, Copenhagen, Denmark. ${ }^{12}$ Department of Infectious Diseases, Hvidovre Hospital, Kettegaards Allé 30, 2650 Hvidovre, Denmark.

\section{Received: 24 May 2016 Accepted: 24 October 2016} Published online: 08 November 2016

\section{References}

1. Markowitz LE, Dunne EF, Saraiya M, Chesson HW, Curtis CR, Gee J, et al. Human papillomavirus vaccination: recommendations of the Advisory Committee on Immunization Practices (ACIP). MMWR Recomm Rep. 2014;63:1-30

2. Juckett G, Hartman-Adams H. Human papillomavirus: clinical manifestations and prevention. Am Fam Physician. 2010;82:1209-13.

3. Walboomers JM, Jacobs MV, Manos MM, Bosch FX, Kummer JA, Shah KV et al. Human papillomavirus is a necessary cause of invasive cervical cancer worldwide. J Pathol. 1999;189:12-9.

4. Cervical Cancer. Estimated Incidence, Mortality and Prevalence Worldwide in 2012. Globocan 2012. WHO. http://globocan.iarc.fr/old/FactSheets/ cancers/cervix-new.asp. Accessed on 21 Dec 2015. 21-12-2015. 21-12-2015. Ref Type: Internet Communication.

5. Chaturvedi AK, Madeleine MM, Biggar RJ, Engels EA. Risk of human papillomavirus-associated cancers among persons with AIDS. J Natl Cancer Inst. 2009:101:1120-30.

6. Brickman C, Palefsky JM. Human papillomavirus in the HIV-infected host: epidemiology and pathogenesis in the antiretroviral Era. Curr HIV/AIDS Rep. 2015;12:6-15.
7. Rocha-Brischiliari SC, Gimenes F, de Abreu AL, Irie MM, Souza RP, Santana $\mathrm{RG}$, et al. Risk factors for cervical HPV infection and genotypes distribution in HIV-infected South Brazilian women. Infect Agent Cancer. 2014;9:6.

8. McKenzie ND, Kobetz EN, Hnatyszyn J, Twiggs LB, Lucci III JA. Women with HIV are more commonly infected with non-16 and -18 high-risk HPV types. Gynecol Oncol. 2010;116:572-7.

9. Denslow SA, Rositch AF, Firnhaber C, Ting J, Smith JS. Incidence and progression of cervical lesions in women with HIV: a systematic global review. Int J STD AIDS. 2014;25:163-77.

10. Ogembo RK, Gona PN, Seymour AJ, Park HS, Bain PA, Maranda L, et al. Prevalence of human papillomavirus genotypes among African women with normal cervical cytology and neoplasia: a systematic review and meta-analysis. PLoS One. 2015;10:e0122488.

11. Bonde J, Rebolj M, Ejegod DM, Preisler S, Lynge E, Rygaard C. HPV prevalence and genotype distribution in a population-based split-sample study of well-screened women using CLART HPV2 human papillomavirus genotype microarray system. BMC Infect Dis. 2014;14:413-4. doi:10.1186/ 1471-2334-14-413

12. Kjaer SK, Munk C, Junge J, Iftner T. Carcinogenic HPV prevalence and age-specific type distribution in 40,382 women with normal cervical cytology, ASCUS/LSIL, HSIL, or cervical cancer: what is the potential for prevention? Cancer Causes Control. 2014;25:179-89.

13. Saraiya M, Unger ER, Thompson TD, Lynch CF, Hernandez BY, Lyu CW et al. US Assessment of HPV Types in Cancers: Implications for Current and 9Valent HPV Vaccines. J Natl Cancer Inst. 2015;107(6).

14. Bruni L, Diaz M, Castellsague X, Ferrer E, Bosch FX, de SS. Cervical human papillomavirus prevalence in 5 continents: meta-analysis of 1 million women with normal cytological findings. J Infect Dis. 2010;202:1789-99.

15. Heard I, Cubie HA, Mesher D, Sasieni P. Characteristics of HPV infection over time in European women who are HIV-1 positive. BJOG. 2013;120:41-9.

16. Heard I. Human papillomavirus, cancer and vaccination. Curr Opin HIV AIDS. 2011:6:297-302.

17. Obel N, Omland LH, Kronborg G, Larsen CS, Pedersen C, Pedersen G, et al. Impact of non-HIV and HIV risk factors on survival in HIV-infected patients on HAART: a population-based nationwide cohort study. PLoS One. 2011;6:e22698

18. Nygard M, Hansen BT, Dillner J, Munk C, Oddsson K, Tryggvadottir L, et al. Targeting human papillomavirus to reduce the burden of cervical, vulvar and vaginal cancer and pre-invasive neoplasia: establishing the baseline for surveillance. PLoS One. 2014;9:e88323.

19. Kjaer SK, Breugelmans G, Munk C, Junge J, Watson M, Iftner T. Populationbased prevalence, type- and age-specific distribution of HPV in women before introduction of an HPV-vaccination program in Denmark. Int J Cancer. 2008;123:1864-70.

20. http:/wwww.dst.dk/da/Statistik/emner/befolkning-og-befolkningsfremskrivning/ folketal.aspx. Accessed on 14 Sept 2012. Webpage in Danish. 14-9-2012. Ref Type: Internet Communication

21. http://www.ssi.dk/Service/Sygdomsleksikon/H/AIDS\%20-\%20HIV.aspx. Accessed on September 14th 2012. Webpage in Danish. 14 Sept 2012. Ref Type: Internet Communication

22. Obel N, Engsig FN, Rasmussen LD, Larsen MV, Omland LH, Sorensen HT. Cohort profile: the Danish HIV cohort study. Int J Epidemiol. 2009;38:1202-6.

23. Thorsteinsson K, Ladelund S, Storgaard M, Ronsholt FF, Johansen IS, Pedersen G, et al. Sexually transmitted infections and use of contraceptives in women living with HIV in Denmark - the SHADE cohort. BMC Infect Dis. 2016;16:81.

24. Screening of cervical cancer - recommendations 2007. The Danish National Board of Health. Available from: http://sundhedsstyrelsen.dk/publ/Publ2007/ PLAN/Kraeft/Anbef_screen_livmoderhals.pdf. Article in Danish. Summary in English. Accessed on 22nd May 2015. 22-5-2015. 22-5-2015. Ref Type: Internet Communication

25. Kaplan JE, Benson C, Holmes KH, Brooks JT, Pau A, Masur H. Guidelines for prevention and treatment of opportunistic infections in HIV-infected adults and adolescents: recommendations from CDC, the National Institutes of Health, and the HIV Medicine Association of the Infectious Diseases Society of America. MMWR Recomm Rep. 2009;58:1-207.

26. Lauritsen JM \& Bruus M. EpiData (version 3.1). A comprehensive tool for validated entry and documentation of data. The EpiData Association, Odense, Denmark, 2003-2005. 11-2-2015. Ref Type: Generic.

27. Pedersen CB. The Danish civil registration system. Scand J Public Health. 2011;39:22-5. 
28. Erichsen R, Lash TL, Hamilton-Dutoit SJ, Bjerregaard B, Vyberg M, Pedersen L. Existing data sources for clinical epidemiology: the Danish National Pathology Registry and Data Bank. Clin Epidemiol. 2010;2:51-6.

29. Solomon D, Davey D, Kurman R, Moriarty A, O'Connor D, Prey M, et al. The 2001 Bethesda System: terminology for reporting results of cervical cytology. JAMA. 2002;287:2114-9.

30. Zeier MD, Botha MH, Engelbrecht S, Machekano RN, Jacobs GB, Isaacs S, et al. Combination antiretroviral therapy reduces the detection risk of cervical human papilloma virus infection in women living with HIV. AIDS. 2015;29:59-66.

31. Marks MA, Gupta S, Liaw KL, Tadesse A, Kim E, Phongnarisorn C, et al. Prevalence and correlates of HPV among women attending family-planning clinics in Thailand. BMC Infect Dis. 2015;15:159.

32. R Core Team (2015). R: A language and environment for statistical computing. R Foundation for Statistical Computing, Vienna, Austria. http:// www.R-project.org/. Accessed 24 June 2015. 24-6-2015. 24-6-2015. Ref Type: Internet Communication

33. Serrano B, Alemany L, Tous S, Bruni L, Clifford GM, Weiss T, et al. Potential impact of a nine-valent vaccine in human papillomavirus related cervical disease. Infect Agent Cancer. 2012;7:38.

34. Tugizov SM, Herrera R, Chin-Hong P, Veluppillai P, Greenspan D, Michael BJ, et al. HIV-associated disruption of mucosal epithelium facilitates paracellular penetration by human papillomavirus. Virology. 2013;446:378-88.

35. Micheletti AM, Dutra VF, Murta EF, Paschoini MC, Silva-Vergara ML, Barbosa e Silva, et al. Cervicovaginal cytological abnormalities in patients with human immunodeficiency virus infection, in relation to disease stage, CD4 cell count and viral load. Diagn Cytopathol. 2009;37:164-9.

36. Rodriguez AC, Schiffman M, Herrero R, Wacholder S, Hildesheim A, Castle $P E$, et al. Rapid clearance of human papillomavirus and implications for clinical focus on persistent infections. J Natl Cancer Inst. 2008;100:513-7.

37. Thorsteinsson $\mathrm{K}$, Ladelund S, Jensen-Fangel S, Katzenstein TL, Johansen IS, Pedersen $\mathrm{G}$, et al. Adherence to the cervical cancer screening program in women living with HIV in Denmark: comparison with the general population. BMC Infect Dis. 2014;14:256.

\section{Submit your next manuscript to BioMed Central and we will help you at every step:}

- We accept pre-submission inquiries

- Our selector tool helps you to find the most relevant journal

- We provide round the clock customer support

- Convenient online submission

- Thorough peer review

- Inclusion in PubMed and all major indexing services

- Maximum visibility for your research

Submit your manuscript at www.biomedcentral.com/submit 